\title{
Rapid Maxillary Expansion- A Narrative Review
}

\author{
Padmini M.N ${ }^{1}$,Lakshmi T ${ }^{2}$,Beenadevi V. $\mathrm{K}^{3}$ \\ ${ }^{1}$ Associate Professor,Department Of Orthodontics And Dentofacialorthopedics, Government Dental College \\ And Research Institute ,Bangaluru, India \\ ${ }^{2}$ Post Graduate Student, Department Of Orthodontics And Dentofacialorthopedics, Government Dental College \\ And Research Institute ,Bangaluru, India \\ ${ }^{3}$ Post Graduate Student, Department Of Orthodontics And Dentofacialorthopedics, Government Dental College \\ And Research Institute ,Bangaluru, India
}

\begin{abstract}
Maxillary expansion is an area where extensive researches have been performed because of its wide range of application.Many designs and modifications have been suggested by various authors since its introduction.The overall goal of this review is to have an insight regarding history,clinical application,effects on maxillary complex andrecent advances in RME.
\end{abstract}

Keyword: $C B C T$, crossbite, orthopaedic correction,rapidmaxillary expansion,transverse deficiency

\section{Introduction}

Malocclusions can manifest in all the three planes that is sagittal,transverse and vertical .Transverse maxillary deficiency is frequently observed in patients who seek orthodontic treatment and is often characterised by unilateral or bilateral crossbite as well as anterior crowding.[1]There are different treatment approaches depending on the skeletal maturity, the amount of desired expansion,and the presence of a concomitant sagittal or vertical problem. In skeletally immature patients, rapid orthopedic palatal expansion (RPE) is a common method of treatment.[2]

Transverse separation of the maxillae through rapid palatal expansion also has the potential for increasing arch perimeter by unravelling crowding in the maxillary arch and helps in correction of disharmonies in thetransverse plane between maxillary and mandibular arches. This procedure which was used as a method of crossbite correction has now has its application for levelling the curve of Wilson, reducing the dark spaces in the buccal corridors and perhaps increase in airway patency[1,4]

The increase in palatal width is the result of growth in the midpalatal suture, which itself undergoes a continual morphological change at the histological level, becomingsuccessively more complicated from birth to the cessation of development.[5] As a result of this maturation process, treatment of transverse discrepancies depends both on the nature of the malocclusion and to a large extent on the age of the patient.[6]RME appliances produce heavy forces that separate the maxillary suture, resulting in maximum skeletal or orthopedic expansion with minimum orthodontic tooth movement.Orthopedic expansion via RME is gained not only by bodily separation of the midpalatal suture, but also by additional buccal rotational force on the maxillary alveolar shelves.

\section{Evolution of RME}

Orthopedic expansion of the maxillary arch (RME) has been accomplished for more than a century. This procedure originated in New York with Emerson .c. Angell in 1860. He placed a screw appliance between the maxillary premolars of a girl aged $14 \frac{1}{2}$ years and widened her arch one quarter inch in 2 weeks. But this could not be supported with radiographs as $\mathrm{x}$ rays were still to be discovered.[8]

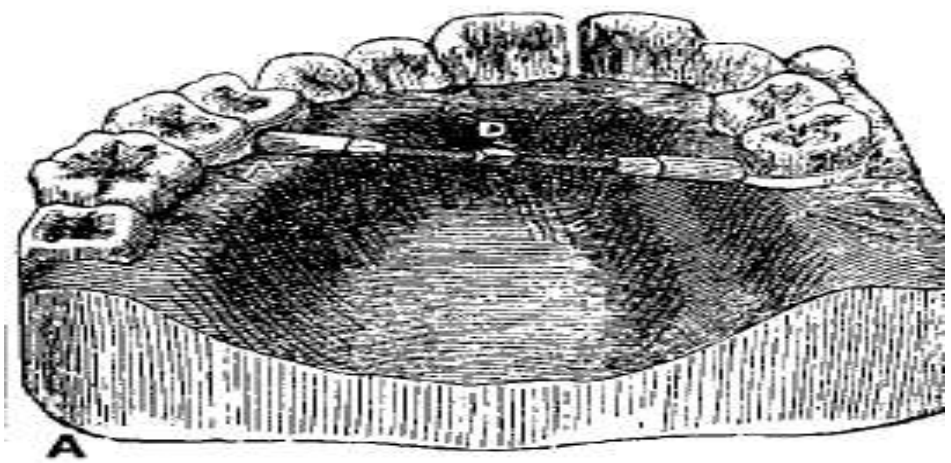

fig 1[8] Angell's expansion appliance 
The procedure was attempted with varying success byFarrar,Goddard,G V Black,Monson, G V I.Brown,Ottolingui, N.M Black,Willis,Barnes, wright Hawley,Pullen,Schroder, Dewey, Huet and Mesnard.Due to wide acceptance of functional concept of development this method of expansion was completely abandoned in the Unites States[9]

Landsbergerwas the first to visualize the skeletal effect of rapid palatal expansion (RPE) with an occlusal radiograph showing the openedmidpalatal suture with the fixed appliance in situ[10].

Korkhaus, probably is responsible for reintroducing this procedure to United State while visiting the department of Orthodontics at the University of Illinois in 1956. But it was not until 1961 in which RME came back on track due to Andrew Hass, who reported a study based on his management of fortyfive patients treated initially by opening the midpalatal suture. Hass tried the RPE technique on pigs and reported among other findings that mandibular dental arch expanded in response to altered functional forces resulting from RPE. [11]

McCraken showed RPE as an integral part of Orthodontic therapy. Wertz confirmed the advantage of RPEin improving nasal air flow in patients with stenosis of nasal airway. He also reported the greatest benefit where stenosis is primarily in anterio-inferior region, while patients with stenosis in the posterior - superior portion of nasal airway did not benefit from RPE. Beiderman expanded Hass's ideas using a tooth borne appliance for maxillary expansion in conjunction with the correction of Class III problems. Timmsconfirmed benefits of RPE for improvement of nasal airway.He found that in all the cases there was a damage to the root surface which was not directed buccally but on mesiobuccal and distobuccal aspect of the roots.[12]

Lines after releasing zygomatic buttress which was the cause of most resistance to RPE, concluded that RPE in adults was possible with use of Corticotomy as an adjunct to mechanotherapy. Kennedy et al stated that Osteotomies through zygomatic buttress, nasomaxillary and pterygomaxillary areas reduce resistance to lateral movements. Hass, in his study on stability of treatment that included RPE demonstrated that 4 to $5 \mathrm{~mm}$ intercanine expansion in the lower arch 9 to $12 \mathrm{~mm}$ expansion in the upper arch remained absolutely stable. [12]

Subtenlyused occlusal bite block with better grasp and overlay of crowns and observed reduction in molar tipping, greater facility to direct forces on to the nasomaxillary complex via root structure itself. Barber and Sims , using fully tooth borne appliance and electron microscopy found cementum and dentin resorption followed by remineralization. Lehman and Hass found that Osteotomy of Zygomaticomaxillary buttress in combination with RPE is the desirable technique for horizontalmaxillary deficiency in adults.[12]

Glassman et al reported performing only lateral maxillary corticotomies and maxillary Hyrax split palate appliance. He confirmed the separation of mid palatal suture by occlusal radiograph and a diastema between maxillary central incisors. Kraut gave osteotomy cuts in the lateral wall of the maxilla combined with pterygomaxillarydysjunction and midpalatal suture separation allowed successful RPE. [12]

Philip M.Nisco showed the control of vertical dimension during rapid palatal expansion using a high pull headgear with chincap.[13] Dale V. H, Peter S,D. Wolf studied the effect of the RME on nasal air way resistance.[14] A. Lindner, C. O. Henrikson. L showed maxillary expansion of unilateral cross-bite in perschool children using RME.[15].Patrick K. Turley used an appliance for correction of class III malocclusion with palatal expansion and custom made protraction headgear. [16]

\section{Clinical applications of RME}

- Patients who have lateral discrepancies that result in either unilateral or bilateral posterior crossbites involving several teeth are candidates for RME. The constriction may be skeletal (narrow maxillary base or wide mandible), dental, or a combination of both skeletal and dental constriction.[12,17]

- Patients with Class III malocclusions, and patients with borderline skeletal and pseudo Class III problems are candidates if they have maxillary constriction or posterior crossbite.[9,12]

- In cleft palate patients with sagittal and transverse discrepancies caused by primary surgeries, RME can be used with a facemask to obtain effective maxillary protaction and improve class III skeletal pattern.[18,19]

- To gain arch length in patients who have moderate maxillary crowding.[4,20]

- Cases of inadequate nasal capacity exhibiting chronic nasal respiratory problems.[9,21]

- According to Bell, the enhanced skeletal response that accompanies RME redirects the developing posterior teeth into normal occlusion and corrects asymmetries of condylar position.[22]

- $\quad$ RME treatment is known to cause relief for the enuretic children, although the long-term success rate is questionable.[23]

- $\quad$ RME is used in the treatment of conductive hearing loss since this procedure aid in improving hearing due to a more normal functioning of the pharyngeal ostia of the Eustachian tubes as a result of the effect of maxillary expansion on the palatal and nasopharyngeal tissues.[24]

\section{Contraindications}

- Noncompliant individuals

- Individuals who have a single tooth crossbite

DOI: 10.9790/0853-1603083542 www.iosrjournals.org
36 | Page 
- Individuals who have anterior open bites, steep mandibular planes, and convex profiles are generally not well suited to RME.

- Individuals who have skeletal asymmetry of the maxilla or mandible, and adults with severe anteroposterior and vertical skeletal discrepancy are not good candidates for RME.[25]

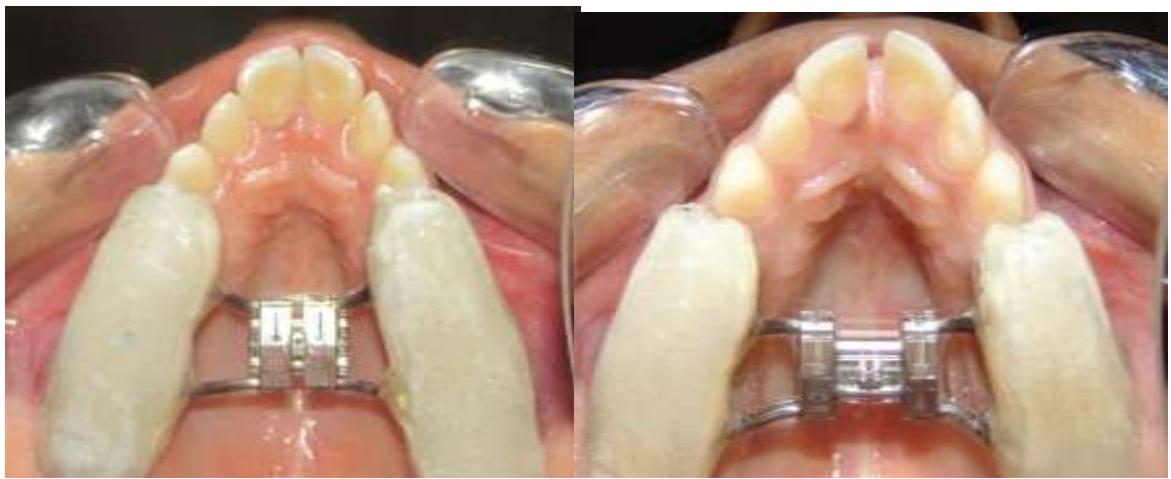

Fig-2 Bonded RME

Appearance of midline diastema

\section{Effects Of RME On Circummaxillary Sutures}

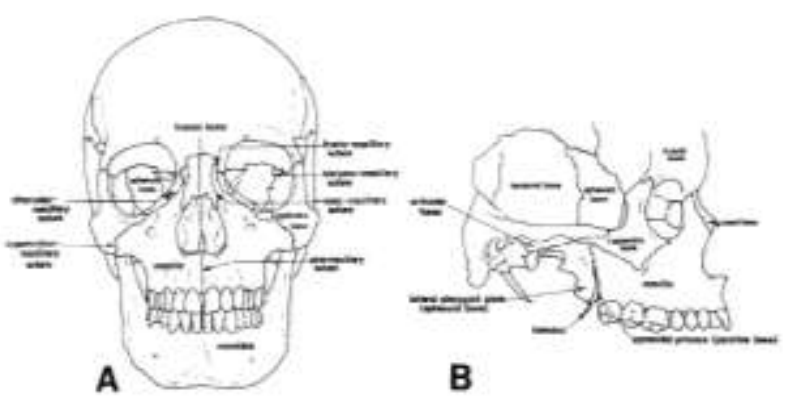

Fig.3Bony articulations of maxilla A. frontal view.B. lateral view [25]

An examination of occlusal films showed that the opening of the midpalatal suture extends through the horizontal plates of the palatine bones, but the distance between the two expanded halves is very narrow. Kudlicin a study on a human dry skull that simulated in vivo response of RME, concluded the following: (1) all craniofacial bones directly articulating with the maxilla were displaced except the sphenoid bone, (2) the cranial base angle remained constant, (3) displacement of the maxillary halves was asymmetric, and (4) the sphenoid bone, not the zygomatic arch: was the main buttress against maxillary expansion. Gardner and Kronman, in a study on RME in rhesus monkeys, found that the lambdoid, parietal and midsagittal sutures of the cranium showed evidence of disorientation, and in one animal these sutures split $1.5 \mathrm{~mm}$. Therefore, RME could affect relatively remote structures and is not limited to the palate. [26]

\section{Effects of RME on the maxillary complex}

The heavy applied pressure acts as an orthopedic force compresses the periodontal ligament, bends the alveolar processes, tips the anchor teeth, and gradually opens the midpalatalsuture.According to "Ekstriim, Henrickson, and Iensen" the mineral content within the suture rose rapidly during the first month after the completion of suture opening which involves tissue injury followed by a hemorrage ,proliferation and repair phenomenon that ultimately leads to regeneration of the suture. From the frontal view, the maxillary suture is found to separate superoinferiorly in a nonparallel manner. The separation is pyramidal in shape with the base of the pyramid located at the oral side of the bone.[25]

\section{Maxillary Halves}

Krebsshowed that the two halves of the maxilla rotated in both the sagittal and frontal planes. Haasand Wertz found the maxilla to be more frequently displaced downward and forward. The final position of the maxilla, after completion of expansion, is unpredictable and it has been reported to return, partially or completely to its original position, In the frontal plane, the fulcrum of rotation for each of the maxillae is said to be approximately at the frontomaxillary suture.[25] 


\section{Palatal Vault}

Fried and Haasreported that there was lowering of palatine processes of the maxilla as a result of the outward tilting of the maxillary halves. Davis and Kronman reported that the palate dome remained at its original height[25]

\section{Alveolar Processes}

Because bone is resilient, lateral bending of the alveolar processes occurs early during RME. Most of the applied forces tend to dissipate within 5 to 6 weeks. After stabilization is terminated, any residual forces in the displaced tissues will act on the alveolar processes resulting in rebound effect. This necessitatesthe need for overcorrection of the constricted dental arches to compensate for the subsequent uprighting of the buccal segment.[25]

\section{Maxillary Anterior Teeth}

Appearance of diastema between the maxillary central incisors is estimated as a active sign ofsuture opening.Following this separation, the incisor crowns converge and establish proximal contact caused by the elastic recoil of the transseptalfibers. This generally takes about 4 months. The maxillary central incisors tend to be extruded relative to the S-N plane and in $76 \%$ of the cases they upright or tip lingually. [25]

\section{Maxillary Posterior Teeth}

With the initial alveolar bending and compression of the periodontal ligament, there is a definite change in the long axis of the posterior teeth. Hicksfound that the angulation between the right and left molars increased from $\mathrm{I}^{\circ}$ to $24^{\circ}$ during expansion. [25]

\section{Palatalmucoperiosteum, Periodontal Tissues, And Root Resorption.}

As the maxillae separate, the palatal mucoperiosteum is stretched. Cotton suggested that the postexpansion angular changes of the maxillary first molars may be related to the stretched fibers of the attached palatal mucosa. Due to sustained heavy forces histologically sterile necrosed areas are seen in the periodontal tissues.. Other investigatorsreported marked buccal root resorption of the anchor teeth during RME and fixed retention. These defects tend to gradually repair.[25]

\section{Efects Of Rme On The Mandible}

Due to disruption of occlusion caused by extrusion and tipping of maxillary posterior teeth along with alveolar bending there is a tendency for the mandible to swing downward and backward which causes the opening of the mandibular plane .[25]

\section{Effects Of Rme On The Mandibular Teeth}

In a study done by Gryson there was a mean increase in the mandibular intermolar width of $0.4 \mathrm{~mm}$ but there was no correlation between the change in mandibular intercanine and intermolar distances with respect to the increase in maxillary intercanine and intermolar distances. RME can influence the mandibular dentition, but it is unpredictable.[25]

\section{Estimating The Needed Expansion}

(1) Measure the distance between the most gingival extension of the buccal grooves on the mandibular first molars or, when the grooves have no distinct terminus on the buccal surface, between points on the grooves located at the middle of the buccal surfaces;

(2) Measure the distance between the tips of the mesiobuccal cusps of the maxillary first molars;

(3) Subtract the mandibular measurement from the maxillary measurement.

The average differences in persons with normal occlusion are $+1.6 \mathrm{~mm}$ for males and $+1.2 \mathrm{~mm}$ for females. The discrepancy between the maxillary and mandibular measurements is a good estimate of how far the maxillary molars must be expanded. One should overexpand the molars 2 to $4 \mathrm{~mm}$ beyond the required distance to allow for the expected postfixation relapse. The expansion screw should provide at least the calculated amount of expansion.[25]

1. Schedule by Timms[27]

\section{Activation schedule}


Patients $</=15$ yrs: 45 degree activation 4 times per day.

Patients $>/=20$ yrs: 45 degree rotation in morning and evening.

2. Schedule by Zimring and Isaacson.[25]

Young growing patients: Two turns per day for 4- 5 days, followed by one turn per day till expansion is achieved.

Non growing adults: Two turns per day for 2 days, then one turn per day for 5-7 days, followed by one turn every alternate day, till expansion is achieved.

3. McNamara and Brudon[4]

once per day activation schedule till expansion is obtained.

4. Semi-rapid Maxillary Expansion (SRME)

As suggested by Iseri activation included two turns per day for the first 5 to 6 days followed by three turns per week for the remainder of the SRME treatment. This stimulates the adaptation process in nasomaxillary complex,thereby minimizing relapse in post-retention period in young and adult patients[28,29]

5.Alternate rapid maxillary expansion and constrictions Repetitive weekly protocol of Alternate rapid maxillary expansions and constrictions(Alt-RAMEC) has theadvantage of displacing the maxilla more anteriorly and disarticulating the circumaxillary sutures more effectively than a singlecourse of RME.[30]

\section{Treatment Timing For RME}

Generally, the suggested time of treatment is between the ages of 6 and 8 years after the maxillary permanent first molars and incisors have erupted. Treating at such an early age is reported to remove factors that inhibit growth and development. Studies reveal that treatment during early mixed dentition or prepubertal age is found to havesignificant and more effective long-term changes at the skeletal level in both maxillary and circummaxillary structures. When RME treatment is performed after the pubertal growth spurt, maxillary adaptations to expansion therapy was more of dentoalveolar than skeletal.[31,32]

\section{Recent Advances In Rme}

As skeletal maturity approaches,bony interdigitation increases and the suture fuses .This leads to difficulty separating the maxilla with orthopaedic force alone. Hence surgical separation of the maxilla along the midline is needed to split the midpalatal suture. A variety of surgical procedures including surgically assisted rapid maxillary expansion and segmental Lefort 1osteotomy have been advocated in the treatment of transverse maxillary deficiency.

\section{Surgically Assissted RME (SARME)}

1.Surgically Assissted RME(SARME)Without Pterygoid Osteotomy (Local Anesthesia)

2.SARME With Pterygoid Osteotomy (General Anesthesia)

Hyrax appliance is placed by the orthodontist prior to surgery. Patient undergoes surgery under local anesthesia or general anesthesia .After surgery Hyrax appliance is activated. Activation time varies according to individual needs.The retention period usually lasts for 6 months to prevent recurrence.[33,34]

In a study on the effect of low level laser therapy (LLLT) on bone regeneration in midpalatal anterior suture after SARME it was found that there was accelerated bone regeneration and increased mineralisation[34]

\section{Microimplant Assisted Rapid Palatal Expansion ( MARPE)}

Recently, microimplant-assisted RPE (MARPE) appliances have become available for treatment of transverse maxillary constriction in older patients that can localize the lateral forces to the midpalatalsuture while minimally using the dentition . [35]

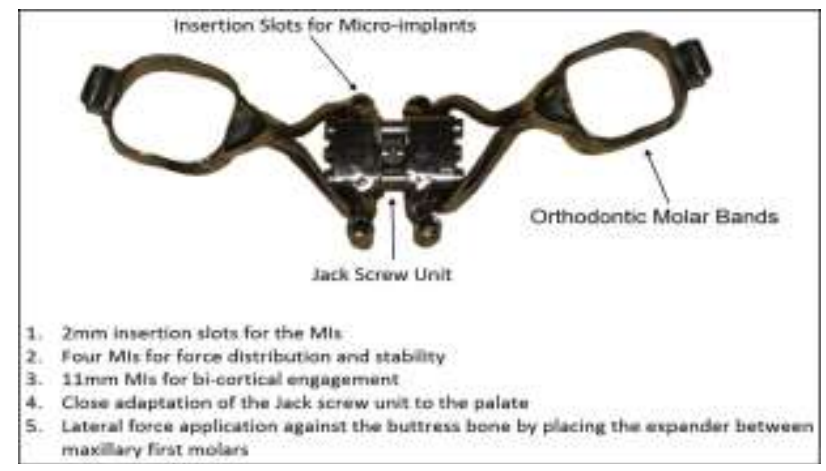

Fig 6[35] 


\section{Retention}

Retention appliance typically used is simple palatal plate that has ball clasps between upper first and second deciduous molars and the upper firstpermanent molars. The patient is instructed to wear the appliance on full time basis. It is advisable to maintain the achieved expansion for atleast one year,if not longer in order to facilitate changes that might be occurring in the mandibular dentition or in the maxillomandibular relationships.[4] Hicks observed that the amount of relapse is related to the method of retention after expansion. With no retention, the relapse can amount to $45 \%$ as compared with $10 \%$ to $23 \%$ with fixed retention and $22 \%$ to $25 \%$ with removable retention. [36]. Bell concluded that slow expansion is less disruptive to tbesuturalsystems. Slow expansion that maintains tissue integrity apparently needs 1 to 3 months of retention, which is significantly shorter than the 3 to 6 months recommended for rapid expansion. Mew advocates a total retention period of $1 \frac{1}{2}$ to 4 years, depending on the extent of expansion.[37]

\section{Recent Studies On RME}

\section{Cbet Studies}

CBCT is an accurate and reliable method for assessing changes associated with RME on nasomaxillary structures.Although the effective radiation dose of CBCT (45-59 $\mu \mathrm{Sv})$ is about 4 to 7 times greater than that of the panoramic radiographs (6.3-13.3 $\mu \mathrm{Sv})$, it is comparable to that of film-based periapical radiographs (14-100 $\mu \mathrm{Sv})$ In addition, when compared with conventional computed tomography (CT) $(314 \mu \mathrm{Sv})$ it produces a much lower effective radiation dose and can produce clear images of highly contrasting structures. So it has been used for 3-dimensional orthodontic diagnosis and assessment of treatment outcomes.[38]CBCT study was done to compare the dentoskeletal effects of tooth tissue borne and tooth borne expanders and results showed both expanders produced similar orthopaedic effects and tooth tissue borne expander produced a greater change in axial inclination of supporting teeth especially in first premolar.[39]CBCT studies on SARPE showed that it has a significant clinical impact on the periodontium.It includes decrease in the buccal alveolar bone thickness( $\mathrm{BABT}$ ) on most teeth ,increase in the palatal alveolar bone thickness, considerable decrease in the buccal alveolar crest level and decrease in the interproximal alveolar crest level on the mesial aspect of both central incisors.[2]

CBCT studies to find immediate and postretention effects of RPE showed significant increase in transverse dimensions at the crown and apex levels of first molars.At the end of active phase of expansion the buccal bone plate thickness of the supporting teeth showed a significant decrease and after a retention period of 6 months there was recovery of both buccal and lingual plate thickness.[40]Banded and bonded hygienic expanders had similar effects on the buccal bone of the maxillary posterior teeth. There is a tendency for vertical buccal bone loss (MBL)that was observed in another CBCT study.[41]

CBCT studies have been done to evaluate the changes in nasomaxillary complex using Hass and Hyrax expanders Both expanders increased the transverse dimension of the skeletal and soft tissue nasomaxillary complex equally. More dental tipping was observedwith the tooth tissue borne expander. Maxillary sinus volume increased between $6 \%$ and $11 \%$ after expansion. No statistical difference was found in the posterior airway volume.[42]

\section{Biochemical Studies}

IL-1beta and betaG are present in the GCF of adolescents, although their level decreases after a strict regimen of plaque control, it increases during orthodontic or orthopedic movement. Moreover, this study demonstrates that both heavy and light forces evoke increased levels of IL-1beta and betaG, stronger forces cause higher levels of inflammatory mediators, and both IL-1beta and betaG respond to direct and indirect application of mechanical force to teeth.[43]

Another study investigated PGE2 in GCF. Both light orthodontic and heavy orthopaedic forces resulted in an increase in PGE2 levels. This was found to be higher in the SARPE cases than in the Trans palatal distraction cases following activation of the screws and may be due to greater dental effects with the SARPE procedure. The low PGE2 levels indicate that using TPD might have prevented unfavourable sequele, such as root resorption, bony dehiscences, and buccal tipping of the anchor teeth[44]

In another study during the retention phase of RME, increases in GCF ALP activity was detected at the tension sites at both 3 and 6 months without clinically relevant tissue inflammation. However, further comprehensive research including other GCF biomarkers and following longterm monitoring protocols are needed to propose that GCF ALP activity is a diagnostic tool for alveolar bone formation of the tension sites during the retention phase of RME.[45] 


\section{Conclusion}

The application of RME has favoured orthodontic fraternity since its advent from 18th century. It has served as a conservative method of gaining space in borderline cases where extraction is controversial.Although many attempts were made for expanding the dental arches RPE is considered to have reasonably good effects on both skeletal and dental tissues. As thetransverse growth of the jaw cease first,all the studies mentioned in the article says that expansion achieved in the earlier ages is more advantageous and stable.Apart from achieving arch expansion it also has its effect on oral, nasal and pharyngeal tissues so as to benefit respiration.Continued research and interest in the field has made room to many newer concepts like studies on biomarkers, implant supported RME and SARPE. Thorough knowledge,keen observation,proper diagnosis and decision can enormously benefit the outcome of treatment.

\section{References}

[1]. McNamara JA, Maxillary transverse deficiency, Am J OrthodDentofacialOrthop 2000;117:567-70.

[2]. Chantal Gauthier, Periodontal effects of surgically assisted rapid palatal expansion evaluated clinically and with cone-beam computerized tomography: 6-month preliminary results ,Am J OrthodDentofacialOrthop 2011;139:S117-28.

[3]. Michael D. Adkins,Arch perimeter changes on rapid palatal expansion.Am j orthoddentofacorthop 1990;97:194-9

[4]. James A Mcnamara,WilliamBrudon,Rapid maxillary expansion appliances, Orthodontics and dentofacial orthopaedics(Needham Press).

[5]. Melsen B,Palatal growth studied on human autopsy material: a histologic microradiographic study. American Journal of Orthodontics, $1975,68: 42-54$.

[6]. Solow B, The dento-alveolar compensatory mechanism: background and clinicalimplications .British Journal of Orthodontic $1980,7: 145-161$

[7]. Birgit Podesser, Evaluation of the effects of rapid maxillary expansion in growing children using computer tomography scanning: a pilot study. European Journal of Orthodontics 29 (2007) 37-44.

[8]. Donald J Timms. The dawn of rapid maxillary expansion. Angle Orthodontist vol 39 (1999).

[9]. Andrew J Hass, Rapid palatal expansion of the maxillary dental arch and nasal cavity by opening midpalatal suture. Angle Orthod,vol.31,no.2.

[10]. Karin Habersack ,High-resolution multislice computerized tomography with multiplanar and 3-dimensional reformation imaging in rapid palatal expansion. Am J OrthodDentofacialOrthop 2007;131:776-8.

[11]. Andrew J Hass ,Treatment of maxillary deficiency by opening the midpalatal suture. Angle Orthod,vol.35,no.3.

[12]. Michel.C.Alpernetal ,Rapid palatal expansion in adults with and without surgery. Angle orthodontist july 1987.

[13]. Philip M Nisco,Control of vertical dimension during rapid palatal expansion using a high-pull chin cup.Am $\mathbf{J}$ OrthodDentofacialOrthop 1987;91,4.

[14]. Dale V. Hartgerink, The effect of rapid maxillary expansion airway resistance, Am J OrthodDentofacialOrthop.1987;92,381-9.

[15]. Lindner, Maxillary Expansion of Unilateral Cross-Bite in Preschool Children. Am J OrthodDentofacialOrthop 1987,September.

[16]. Turley P K,Orthopedic correction of Class III malocclusion with palatal expansion and custom protraction headgear.Journal Of Clinical Orthodontics,1988,22,5,314-25.

[17]. Mugesandikcioglu,Skeletal and dental changes after maxillary expansion in the mixed dentition.Am J Orthod 1997;111:321-7.

[18]. Robert $\mathrm{j}$ isaacsonetal,Some effects of rapid maxillary expansion in cleft lip and palate patients.Angle orthod 1964.

[19]. Omar Gabriel da Silva Filhoetal, Rapid Maxillary Expansion After Secondary Alveolar Bone Grafting in Patients With Alveolar Cleft The Cleft Palate. Craniofacial Journal. 2009;46(3):331-338.

[20]. Sabrina Mutinelli et al,Anchorage onto deciduous teeth: effectiveness of early rapid maxillary expansion in increasing dental arch dimension and improving anterior crowding. Progress in Orthodontics (2015) 16:22.

[21]. Robert A. Wertz et-al,Changes in Nasal Air Flow Incident to Rapid Maxillary Expansion. Angle orthodontist ,vol 52,no 9.

[22]. Bell RA. A review of maxillary expansion in relation to rate of expansion and patient's age. AM J Orthod 1982:81:32-7.

[23]. SerdarUsumezaD ,Effect of Rapid Maxillary Expansion on Nocturnal Enuresis. Angle Orthod 2003;73:532-538.

[24]. Theodore Laptook, Williston Park, N. Y,Conductive hearing loss and rapid maxillary expansion Am J Orthod1981Vol 80 No.3,325331

[25]. Samir EBishara ,Maxillary expansion :clinical implications Am J Orthoddentofacorthop 1987,91, 3-14

[26]. Wertz RA, Skeletal and dental changes accompanying rapid midpalatal suture opening. Am J Orthod1970;58:41-66.

[27]. Donald J. TimmsRapid maxillary Expansion (Quintessence publishers)

[28]. Iseri H, Ozsoy S, Semirapid maxillary expansion--a study of long-term transverse effects in older adolescents and adults. Angle Orthod ,2004,74(1):71-78

[29]. SabriiihanRamoglu ,Maxillary expansion in mixed dentition rapid or semi rapid ,Euro JOrthod,2010

[30]. Eric jein-weinliou,wen-chingtsai ,A New Protocol for Maxillary Protraction in Cleft Patients:Repetitive Weekly Protocol of Alternate Rapid Maxillary Expansions and Constrictions.

[31]. TizianoBaccetti ,Treatment timing for rapid maxillary expansion.Angle Orthod 2001,71,343-350.

[32]. Lorenzo Franchi, TizianoBaccetti,Postpubertal assessment of treatment timing for maxillary expansion and protraction therapy followed by fixed appliances. Am J OrthodDentofacialOrthop 2004;126:555-68

[33]. Bruno Ramos etal ,Orthodontic or surgically assisted rapid maxillary expansion .Oral And MaxillofacSurg 2009,13,123-137.

[34]. PierangeloAngeletti ,Effect of low level laser therapy on bone regeneration in midpalatal anterior suture after surgically assisted rapid maxillary expansion. OOOOE 2010,109,e38-e46.

[35]. Chuck Carlson,a Jay Sung,b Ryan W. McComb ,Microimplant-assisted rapid palatal expansion appliance to orthopedically correct transverse maxillary deficiency in an adult. Am J OrthodDentofacialOrthop 2016;149:716-28

[36]. Preston Hicks,Slow maxillary expansionA clinical study of the skeletal versus dentalresponse to low-magnitude force,American journal of orthodontics, Vol 73, No 2,1978.

[37]. John Mew,Relapse following maxillary expansion A study of twenty-five consecutive cases. Am J Orthod,vol 83,No 1 .

[38]. SukovicP,Cone beam computed tomography in craniofacial imaging. OrthodCraniofac Res 2003;6(Suppl 1):31-6.

[39]. Daniela G. Gariba; Jose' Fernandoetal,Rapid Maxillary Expansion-Tooth Tissue-Borne Versus Tooth-Borne Expanders: A Computed Tomography Evaluation of Dentoskeletal Effects ;Angle Orthod 2005;75:548-557 
[40]. FabianaBallanti ,Immediate and post retention effects of rapid maxillary expansion investigated by computed tomography in growing patients. Angle orthod 2009,79,24-29.

[41]. Valmy P-Kulbershaetal,CBCT assessment of alveolar buccal bone level after RME. Angle Orthod. 2013;83:110-116.

[42]. ValmyPangrazio-Kulbersha; Paul Wine etal ,Cone beam computed tomography evaluation of changes in the naso-maxillary complex associated with two types of maxillary expander.Angle Orthod. 2012;82:448-457.

[43]. Tzannetou S1, Efstratiadis S,Comparison of levels of inflammatory mediators IL-1beta and betaG in gingival crevicular fluid from molars, premolars, and incisors during rapid palatal expansion.Am J OrthodDentofacialOrthop. 2008;133(5):699-707.

[44]. EmelSari ,OnurKadioglu ,Prostaglandin E2 levels in gingival crevicular fluid during tooth- and bone-borne expansion .European Journal of Orthodontics 32 (2010) 336-341.

[45]. Giuseppe Perinetti, FabriziaD'Apuzzoetal,Gingival crevicular fluid alkaline phosphate activity during the retention phase of maxillary expansion in prepubertal subjects: A split-mouth longitudinal study,Am J OrthodDentofacialOrthop 2015;148:90-6. 